\title{
28 Research Suare \\ FRET-FISH probes chromatin compaction at individual genomic loci in single cells
}

\section{Ana Mota}

Karolinska Institute https://orcid.org/0000-0001-8592-9764

\section{Erik Wernersson}

Karolinska Institutet https://orcid.org/0000-0003-4778-1660

\section{Xiaoze Li-Wang}

Karolinska Institutet, SciLifeLab

\section{Katarina Gradin}

Karolinska Institutet, SciLifeLab

\section{Nicola Crosetto}

Karolinska Institute https://orcid.org/0000-0002-3019-6978

Magda Bienko ( $\nabla$ magda.bienko@scilifelab.se)

Karolinska Institutet, SciLifeLab https://orcid.org/0000-0002-6499-9082

\section{Article}

Keywords:

Posted Date: December 8th, 2021

DOI: https://doi.org/10.21203/rs.3.rs-1130822/v1

License: (c) (i) This work is licensed under a Creative Commons Attribution 4.0 International License. Read Full License

Version of Record: A version of this preprint was published at Nature Communications on November 5th, 2022. See the published version at https://doi.org/10.1038/s41467-022-34183-y. 


\section{Abstract}

The density or compaction of chromatin throughout the cell nucleus is a key biophysical property that influences DNA replication, transcription, and repair. Chromatin accessibility is often used as a proxy for chromatin compaction or density, however it is not clear how these two properties relate to each other, given the lack of tools for directly probing compaction at defined genomic loci. To fill in this gap, here we developed FRET-FISH, a microscopy-based method combining fluorescence resonance energy transference (FRET) with DNA fluorescence in situ hybridization (FISH) to probe chromatin compaction at selected loci in single cells. We optimized FRET-FISH by testing different probe designs in situ in fixed cells, readily detecting FRET generated by DNA FISH probes. To validate FRET-FISH, we compared it with ATAC-seq and $\mathrm{Hi}-\mathrm{C}$, demonstrating that local chromatin compaction and accessibility are strongly correlated and that the frequency of intra-genic contacts measured by Hi-C may be an even better proxy for local chromatin density. To further validate FRET-FISH, we showed that it can detect expected differences in chromatin compaction along the nuclear radius, with peripheral loci being more compacted and central ones less compacted. Lastly, we assessed the sensitivity of FRET-FISH, demonstrating its ability to reproducibly detect differences in chromatin density (i) upon treatment of cells with drugs that perturb global chromatin condensation; (ii) during prolonged cell culture; and (iii) in different phases of the cell cycle. We conclude that FRET-FISH is a robust tool for probing chromatin compaction at selected loci in single cells and for studying inter-allelic and cell-to-cell variability in chromatin density.

\section{Introduction}

Three major types of chromatin have been described in the nucleus of mammalian cells: active, repressed and inactive ${ }^{1-3}$. Active chromatin corresponds to euchromatin described in early electron microscopy studies and is characterized by active gene expression, histone depletion around transcriptional start sites, and histone marks promoting the recruitment and function of transcriptional complexes, such as histone $\mathrm{H} 3$ acetylated on lysine 27 (H3K27ac) or tri-methylated on lysine 4 (H3K4me3) ${ }^{4}$. Repressed chromatin marks genomic regions that are not being actively transcribed in a particular cell type and is enriched in histone $\mathrm{H} 3$ tri-methylated on lysine 27 (H3K27me3) ${ }^{5}$. In contrast, inactive chromatin encompasses genomic regions that are presumably never transcribed, such as peri-centromeric regions, and is decorated by heterochromatic proteins like heterochromatin protein 1 (HP1) or histone H3 methylated on lysine 9 (H3K9me $)^{6}$.

It is generally accepted that different chromatin types correspond to different levels of compaction or density defined as chromatin mass per unit volume. Traditionally, gross differences in chromatin density have been revealed by fluorescence microscopy using DNA intercalating fluorescent dyes, such as 4',6diamidino-2-phenylindole (DAPI), which allow distinguishing between highly compacted and looser chromatin regions ${ }^{7}$. However, the use of DNA intercalators only does not allow direct measurements of chromatin compaction at defined loci and can be biased by the preferential binding of DNA intercalators to certain genomic sequences, such as DAPI binding stronger to AT-rich DNA stretches ${ }^{8}$. To avoid such 
potential biases, several alternative approaches have been deployed. For example, a photo-switchable protein fused to histone H2B (H2B-PATagRFP) was applied in combination with super-resolution microscopy, revealing that inactive chromatin has a lower chromatin compaction and mobility compared to active chromatin ${ }^{9}$. Histone $\mathrm{H} 2 \mathrm{~B}$ fusion to a fluorescent protein was also used to reveal substantial heterogeneity in chromatin compaction across the cell nucleus, by combining fluorescence lifetime imaging (FLIM) and fluorescence energy transfer (FRET) ${ }^{10,11}$. One limitation of these approaches is that they are blind to the underlying DNA sequence and thus are not informative about the local chromatin compaction at specific loci. Instead, DNA fluorescence in situ hybridization (FISH) allows visualizing selected DNA loci, however this technique has not been used to systematically probe for chromatin compaction.

Following the advent of massively parallel sequencing technologies, several methods have been developed to probe the accessibility of chromatin genome wide, including DNase-seq ${ }^{12}$, MNase-seq ${ }^{13}$ and ATAC-seq ${ }^{14}$. These methods do not measure chromatin compaction directly, but rather probe the accessibility of the linear genome to DNA nucleases (DNase-seq), restriction enzymes (MNase-seq) or transposases (ATAC-seq). Although it is assumed that chromatin accessibility and density are inversely correlated, the exact relationship between these two biophysical properties remains unknown. For instance, a locus that is densely coated by various proteins might be inaccessible to nucleases or transposases, yet its chromatin might occupy a relatively large volume and thus have relatively low density. Thus, new tools that can probe chromatin compaction or density directly at defined genomic loci are needed to gain deeper insights into the physical structure of chromatin in the nucleus.

Towards this goal, here we present a novel microscopy-based method integrating DNA FISH with FRET to measure chromatin compaction at individual gene loci in single cells (FRET-FISH). We show that the compaction at a given locus can be detected by FRET-FISH as a difference in FRET efficiency, when targeting the region with carefully designed and empirically tested oligonucleotide (oligo) FISH probes consisting of oligos with alternating FRET donor and acceptor dyes. We demonstrate that chromatin density measured by FRET-FISH strongly correlates with chromatin accessibility measured by ATAC-seq and even stronger with chromosome contact frequencies of the same loci measured by Hi-C ${ }^{15}$. Lastly, we show that FRET-FISH can detect changes in local chromatin compaction in cells treated with drugs that induce global changes in chromatin condensation, in cultured cells at increasing passage numbers, as well as in cells in different phases of the cell division cycle. We conclude that FRET-FISH is a sensitive assay that can be used to measure chromatin density at selected loci and to assess inter-allelic and cellto-cell differences in local chromatin compaction within a cell population.

\section{Results}

\section{Implementation of FRET-FISH}


FRET-FISH is based on the hybridization-in fixed cells-of oligonucleotide (oligo) DNA FISH probes coupled to two fluorescent dyes with overlapping spectra targeting two proximal DNA sequences, so that if the two sequences are closer than the Förster distance, $R_{0}$, FRET is detected (Fig. 1a). We reasoned that a FISH probe consisting of multiple oligos carrying alternating FRET donor (D) and acceptor (A) dyes, targeting a given genomic locus, should enable probing chromatin compaction at that locus by measuring FRET efficiency. Because FRET efficiency is influenced by the dipole orientation and the molecular distance between $\mathrm{D}$ and A dyes, we empirically tested three different FRET-FISH probe designs, all targeting a region of 20 kilobases $(\mathrm{kb})$ encompassing the human $M Y C$ gene locus, to check which probe design yields the highest FRET efficiency (Fig. 1b and Supplementary Table 1). The first design is essentially identical to the iFISH probe design that we previously described ${ }^{16}$ and consists of primary oligos with a target $(T)$ sequence complementary to the genomic DNA target (60 nucleotides (nt) instead of $40 \mathrm{nt}$ as in iFISH) and left ( $\mathrm{L}$ ) and right (R) adapter sequences that are needed for PCR during the production of the probes and serve as docking sites for fluorophore-conjugated detection oligos ( $L^{*}$ and $R^{\star}$, respectively) (Fig. 1b, Design 1). In the second design, the $L$ and $R$ sequences of each primary oligo are extended with a left and right stabilizing sequence (LSS and RSS, respectively), where the $3^{\prime} 6 \mathrm{nt}$ of the RSS in one oligo are complementary to the $5^{\prime} 6 \mathrm{nt}$ of the LSS in the next primary oligo along the linear genomic target (Fig. 1b, Design 2). We reasoned that this design should stabilize the proximity between $D$ and $A$ dyes, thus enhancing FRET efficiency. Lastly, in the third design, the stabilizing sequence is added to the $L^{*}$ and $R^{*}$ detection oligos, so that the $3^{\prime} 6 \mathrm{nt}$ of an $L^{*}$ oligo can anneal to the $5^{\prime} 6 \mathrm{nt}$ of the $\mathrm{R}^{*}$ oligo bound to the next primary oligo along the linear genomic target (Fig. 1b, Design 3). In all three cases, we designed each probe to contain 134 primary D-A oligo pairs with a minimum distance of $5 \mathrm{nt}$ between the 3 ' of the T sequence of a primary D oligo and the $5^{\prime}$ of the $T$ sequence of the next primary A oligo along the linear genomic target. We used Cy3 and Cy 5 as D and A dyes, respectively, since they are widely used in FRET experiments due their relatively high brightness and lower price compared to other fluorophores.

To test each probe design, we hybridized HAP1 human chronic myeloid leukemia cells inside customdesigned 9-well silicone-coated coverslips to minimize technical variability between samples and to compare all three probe designs within the same experiment (Supplementary Fig. 1a and Methods). We calculated the FRET efficiency by dividing the signal intensity detected in the A channel (Cy3 excitation and Cy5 emission) by the sum of the signal intensities in the D (Cy3 excitation and Cy3 emission) and $A$ channels (Methods). In two independent experiments, all the three designs produced readily detectable FRET signals, with Design 3 yielding the highest FRET efficiency (39.5\% $\pm 6.8 \%$, mean \pm s.d.), most likely because the presence of complementary annealing sequences in the detection oligos stabilizes the primary D-A oligo pairs that are in physical proximity (Fig. 1c and Supplementary Fig. 1b). Surprisingly, in both replicate experiments, Design 2 yielded the lowest FRET efficiency $(24.8 \% \pm 5.2 \%$, mean \pm s.d.), possibly because the stabilization sequence in the primary oligos hindered the energy transfer between the $D$ and $A$ dyes or because it acted as a quencher (Fig. 1c and Supplementary Fig. 1b). Independently of the probe design, the FRET efficiency was consistently lower in control samples in which only D or A primary oligos were hybridized, while detection oligos targeting both were included, demonstrating the specificity of our approach (Fig. 1c and Supplementary Fig. 1b). Importantly, the FRET efficiency 
distributions were similar between controls, indicating that the influence of cross-excitation was similar in all the designs tested. We obtained similar results using 6-well chambered coverslips where two designs were compared side-by-side in each experiment (Supplementary Fig. 1c-f). Altogether, these results demonstrate that proximity between in situ hybridized oligos carrying alternating FRET acceptor and donor dyes can be detected by measuring FRET efficiency in fixed cells.

\section{Optimization of FRET-FISH probe design to measure local chromatin compaction}

Prompted by these results, we then sought to further optimize the FRET-FISH probe design to measure local chromatin density at selected genomic loci. We reasoned that the three probe designs that we tested might not allow detecting changes in chromatin compaction, given that in those probes $D$ and $A$ primary oligos bind very close along the linear genome. Instead, we designed probes consisting of $D$ and $A$ primary oligos separated by larger linear genomic distances, so that their physical distance in the nucleus would be sensitive to the extent of local chromatin compactness. We tested three different spacing (S) distances (50,150, and $300 \mathrm{nt}$ ) between consecutive oligos, as in a previous study on purified nucleosomes it was shown that FRET dyes positioned at similar distances yield detectable FRET signals ${ }^{17}$. We also modified the probe design so that each probe would consist of alternating groups $(\mathrm{G})$ of 1,2 or $4 \mathrm{D}$ oligos followed by groups of 1,2 or 4 A oligos (Fig. 1d-i). We reasoned that, since the oligo hybridization efficiency in DNA FISH most likely does not reach $100 \%$, having consecutive groups of multiple $D$ and $A$ oligos would maximize the chances of multiple D-A pairs to be in sufficient spatial proximity to yield a FRET signal. We named the different designs G1-S50, G1-S150, G2-S50, G2-S300, G4S50, and G4-S300 (Fig. 1d-i). As a proof-of-concept, we targeted the mouse Ogt gene locus located on chromosome (chr) $\mathrm{X}$, by hybridizing female mouse embryonic fibroblasts (MEFs), reasoning that we might detect differences in chromatin compaction since this locus is known to escape female $X$ chromosome inactivation, albeit at a low $(\sim 6 \%)$ frequency ${ }^{18}$. All the six probe designs yielded detectable FRET signals in dozens of single cells analysed, however the distributions of the FRET efficiency differed depending on the probe design (Fig. 1d-i). Design G1-S150 and G2-S50 showed a major FRET efficiency peak on the right, most likely corresponding to Ogt alleles within compacted chromatin, flanked by a leftward lower FRET efficiency tail presumably corresponding to Ogt alleles in a less compacted state (Fig. 1d, e). Of note, the FRET efficiency consistently peaked around $40 \%$ in all the probe designs tested, and this finding was recapitulated in a different cell line (NIH3T3 fibroblasts) (Supplementary Fig. 2a-f). We also examined the distribution of FRET acceptor intensities across all the probe designs and did not detect any major differences (Supplementary Fig. 2g-l), demonstrating that the ability of FRET-FISH probes to detect chromatin compaction differences at the targeted loci depends on the generation of FRET. Lastly, we tested a different pair of FRET dyes, AlexaFluor 488 (AF488) and AlexaFluor 594 (AF594), which are characterized by higher quantum yield and lower fluorescence signal degradation over time compared to Cy3 and Cy5. Using the same Ogt probe design, G1-S150, together with AF488 and AF594 dyes resulted in clearly detectable FRET with reduced crosstalk and bleed-through compared to the use of Cy3 and Cy5 (Fig. 1j and Supplementary Fig. 3). Moreover, the same probe design and dye 
combination yielded two clearly distinct FRET efficiency peaks indicative of different underlying chromatin compaction states (Fig. 1k). Therefore, we adopted the G1-S150 probe design and AF488 (FRET donor) and AF594 (FRET acceptor) dyes as standard FRET-FISH settings for all subsequent experiments.

\section{FRET-FISH validation}

Next, we sought to validate FRET-FISH by comparing chromatin compaction measurements at selected loci with chromatin accessibility measured at the same loci by ATAC-seq ${ }^{14}$. To this end, we designed FRET-FISH probes against six genes (Atp2b3, Ddx3x,Kdm5c, Magix, Pbdc1, and Tent5d) on mouse chrX, including three genes (Atp2b3, Magix, and Tent5d) that are constitutively inactivated on one chrX copy and three genes $(D d x 3 x, K d m 5 c$, and $P b d c 1)$ that frequently escape inactivation ${ }^{18}$ (Supplementary Table 1). In three independent experiments, we measured FRET at each of the six targeted loci in female MEFs, which yielded reproducible FRET efficiency bimodal distributions, indicating that these loci most likely exist in two distinct chromatin compaction states (Fig. 2a-f and Supplementary Fig. 4a-r). Of note, the FRET efficiency distributions were unique to each gene and there was no obvious difference between constitutive active and escapee genes.

We then retrieved ATAC-seq read counts from MEFs, matching all the six gene loci probed by FRET-FISH (Supplementary Table 1 and Supplementary Methods). We compared the mean FRET efficiency measured at each locus with the corresponding ATAC-seq read counts and found an inverse correlation between the two metrics (Pearson's correlation coefficient, PCC: -0.75$)$, demonstrating that local chromatin accessibility and density are indeed associated (Fig. 2g). To further validate FRET-FISH, we assessed whether local chromatin compaction measured by FRET-FISH correlates with contact frequencies measured by $\mathrm{Hi}-\mathrm{C}^{15}$ at the same loci. To this end, we retrieved publicly available $\mathrm{Hi}-\mathrm{C}$ data from $\mathrm{MEFs}^{19}$ and correlated the total number of contacts within the genomic windows encompassing the six gene loci probed by FRET-FISH with the corresponding mean FRET efficiencies measured by FRETFISH (Supplementary Table 2 and Supplementary Methods). The correlation between FRET-FISH and Hi-C was considerably stronger (PCC: -0.98) than between FRET-FISH and ATAC-seq, suggesting that Hi-C can be used to probe chromatin compaction genome wide (Fig. 2h). While FRET-FISH and ATAC-seq showed poor concordance for the $D d x 3 x$ gene locus, we instead observed a high agreement between FRET-FISH and $\mathrm{Hi}-\mathrm{C}$ at this locus (Fig. 2h). Importantly, the correlation between FRET-FISH and Hi-C decreased when we only considered $\mathrm{Hi}-\mathrm{C}$ contacts over short distances $(<5 \mathrm{~kb})$ within the genomic windows encompassing the six genes probed by FRET-FISH (Fig. 2i). This suggests that FRET is not only generated by consecutive $D$ and A oligos in our FRET-FISH probes, but also by D-A pairs separated by higher genomic distances that are in physical proximity in the nucleus. Of note, the correlation between ATACseq and Hi-C (PCC: 0.78) was, in absolute terms, lower than between FRET-FISH and either method (Supplementary Fig. 4s), suggesting that Hi-C might be better suited than ATAC-seq to probe chromatin density genome wide. Altogether, these results demonstrate that FRET-FISH is a valid method allowing reproducible measurements of local chromatin compaction at selected genomic loci, and that global and local chromatin accessibility and density are closely intertwined. 


\section{FRET-FISH detects chromatin compaction changes along the nuclear radius}

To further validate FRET-FISH, we examined whether our method can also distinguish between different chromatin density states along the nuclear radius. In mammalian cells, the genome is radially organized and is characterized by distinct radial patterns of different chromatin types and genomic features ${ }^{20,21}$. In particular, highly condensed heterochromatin is enriched in proximity to the nuclear lamina and around nucleoli ${ }^{21}$. We therefore reasoned that FRET-FISH should yield different FRET efficiency distributions for the same locus, depending on where the locus is radially positioned in a cell. To test this, we assessed the FRET efficiency distribution in four arbitrary concentric nuclear layers drawn in 2D segmented nuclei (Supplementary Methods). At three loci examined (Apt2b3, Kmd5c and Magix), the FRET efficiency progressively decreased from the nuclear periphery inwards, in line with our expectation that a locus positioned at the nuclear periphery would have a more compact chromatin compared to the same locus positioned more centrally (Fig. 2j-I). These results are consistent with a radial gradient model of genome organization ${ }^{21}$ and further highlight the ability of FRET-FISH to detect local chromatin density differences with single-allele resolution.

\section{FRET-FISH can detect local effects of global chromatin condensation changes}

Next, we sought to test whether FRET-FISH could detect local changes in chromatin density associated with global changes in chromatin condensation. To this end, we treated female MEFs with a combination of sodium azide and 2-deoxy-d-glucose, which causes intracellular ATP depletion and, in turn, increases the intracellular pool of polyamines and divalent cations that neutralize the negative charges on DNA, leading to chromatin condensation ${ }^{22}$ (Supplementary Methods). The same treatment also results in drastic reduction of gene expression ${ }^{23}$. To confirm this effect in our experimental setup, we quantified by fluorescence microscopy nascent transcripts, in which we incorporated the fluorescent uridine analog 5ethynyluridine (EU) and observed a drastic reduction in the total amount of nuclear fluorescence upon treatment of MEFs with sodium azide and 2-deoxy-d-glucose (Supplementary Fig. 5a, b). To test whether these drastic global changes in chromatin condensation are reflected at the level of individual genes, we performed FRET-FISH in female MEFs that were either treated with sodium azide and 2-deoxy-d-glucose or not, using four of the six probes targeting different genes (Atp2b3, Kdm5c, Magix and Pbdc1) on mouse chrX (Supplementary Table 1). In two independent experiments, ATP-depleted cells showed a significantly higher FRET efficiency at all the four gene loci examined (Fig. 3a-d and Supplementary Fig. 5c-f), demonstrating that FRET-FISH can reproducibly detect local changes in chromatin density that mirror global changes in chromatin condensation.

To further assess the ability of FRET-FISH to detect local changes in chromatin compaction resulting from global changes in chromatin condensation, we performed FRET-FISH in female MEFs cultured for varying passage numbers, which we hypothesized might result in increased global chromatin compaction 
based on previous studies ${ }^{24-26}$. Indeed, MEFs that underwent more than 10 passages showed a significantly higher $\left(P=1.8 \times 10^{-70}\right.$, Wilcoxon test, two-tailed) global chromatin compaction-as determined based on the mean fluorescence intensity per nucleus of the DNA intercalator Hoechst 33342 -compared to cells that underwent less passages (Supplementary Fig. $\mathbf{5 g}$ and Supplementary Methods). We then assessed whether FRET-FISH would detect a corresponding increase in local compaction at the level of individual gene loci. In two independent experiments, the height of the right peak of the FRET efficiency bimodal distributions measured at the Atp2b3,Kdm5c, and Magix loci (which most likely corresponds to a higher local compaction state) progressively increased upon prolonged cell passaging, mirroring the global increase in chromatin condensation (Fig. 3e-j and Supplementary Fig. 5h-m). Altogether, these results confirm that FRET-FISH can reproducibly detect local chromatin density changes, with single-allele resolution.

\section{FRET-FISH detects chromatin compaction changes during the cell cycle}

Lastly, we tested whether FRET-FISH could detect changes in local chromatin density in different phases of the cell division cycle, during which chromatin undergoes dramatic condensation changes reaching the highest compaction during mitosis ${ }^{27}$. To this end, we classified the same cells, in which we previously measured the compaction at the Atp2b3,Kdm5c, and Magix loci, as either G1 or non-G1 based on the fluorescence intensity of the DNA intercalator dye, Hoechst 33342 in the cell nucleus (Supplementary Fig. 6a and Supplementary Methods). In the case of Magix, we also managed to identify mitotic cells with detectable FRET signals. As expected, the FRET efficiency measured by FRET-FISH in these cells was significantly higher $(P=0.008$, Wilcoxon test, two-tailed) compared to cells in other phases

(Supplementary Fig. 6b). Notably, for all the three genes tested in two independent experiments, the FRET efficiency was substantially lower in non-G1 compared to G1 cells, which was reflected in higher left peaks in the bimodal FRET efficiency distributions in non-G1 cells (Fig. 3k-s and Supplementary Fig. 6ch). These results are consistent with prior observations based on the ATAC-see method, which allows visualizing global DNA accessibility in single cells ${ }^{28}$. We then further separated $\mathrm{G} 1$ cells into Hoechst ${ }^{H i g h}$ (top quantile) and Hoechst ${ }^{\text {Low }}$ (all remaining G1 cells) and found that the FRET efficiency measured at

the same three loci was significantly higher in Hoechst ${ }^{\text {High }}$ cells, which most likely represent cells that just exited mitosis (Fig. 3k-m). These results further highlight the sensitivity of FRET-FISH and its ability to reproducibly detect local DNA density differences, at the level of individual alleles.

\section{Discussion}

We have developed a method, FRET-FISH, which combines the specificity of DNA FISH with the sensitivity of FRET to probe chromatin compaction at selected genomic loci. FRET measurements are notoriously challenging in fixed cells and a combination of FRET and DNA FISH has not been described before. We demonstrate that the FRET efficiency can be modulated by rationally designing different types of DNA FISH probes, with the highest efficiency obtained with probes in which detection oligos binding to 
consecutive target-specific oligos carry extra 6 nt sequences which stabilize the proximity between the FRET donor and acceptor dyes coupled to them (see Design 3 in Fig. 1b). Although this FRET-FISH probe design allowed us to successfully monitor local chromatin density changes at various genomic loci, we cannot exclude that further probe design optimization might result in an even higher FRET efficiency and increased assay sensitivity.

Compared to the iFISH probes that we previously described ${ }^{16}$, FRET-FISH probes contain more oligos ( $400 \mathrm{vs}$. 96 typically used in iFISH) to maximize the likelihood that a sufficient number of FRET donor and acceptor dyes are found in close proximity and thus can generate a detectable signal. As we have shown here, FRET-FISH probes containing 450 oligos can be successfully used to detect local changes in chromatin compaction within a region of $\sim 100 \mathrm{~kb}$. FRET-FISH probes containing more oligos targeting larger genomic regions could be used, for example, to probe for chromatin density across individual topologically associating domains (TADs) ${ }^{29}$. FRET-FISH probes of any size can be easily designed using our freely available scripts (see Code Availability) and produced from synthetic oligopools using the iFISH pipeline that we previously described ${ }^{16}$.

We have observed a strong dependency of the FRET efficiency on the cell cycle phase as well as on the passage number of the cells analyzed by FRET-FISH. Indeed, culturing of MEF cells for more than 10 passages considerably increased the fraction of alleles that FRET-FISH detected in a more compacted state, indicating that differences in cell culturing conditions might result in very different measurements of chromatin compaction. Hence, we recommend to carefully control for possible passage number and cell cycle effects when interpreting the results of FRET-FISH experiments performed in cultured cells.

By comparing FRET-FISH measurements with ATAC-seq data available for the same cell type, we have shown that local chromatin density is inversely correlated with chromatin accessibility at the same loci. However, since we have only probed a small number (6) of loci on a single chromosome (chrX), we cannot establish whether the same relationship is generalizable to all chromosomes and loci. For example, chromatin might be inaccessible at certain loci because of the presence of multiple proteins and/or protein complexes bound to DNA, yet chromatin at the same locus might have a relaxed and open conformation. Indeed, the $D d x 3 x$ and Magix loci, which we have probed by FRET-FISH, provide an interesting example of two loci with very similar accessibility, yet quite different compaction. We anticipate that application of FRET-FISH to probe multiple loci on different chromosomes or embedded in different chromatin types will provide a comprehensive portrait of the relationship between chromatin compaction and accessibility and how this varies between cell types.

Beyond studying the relationship between chromatin density and accessibility, FRET-FISH could also be applied to study enhancer-promoter contacts or chromatin loop organization in single cells, without the need to rely on super-resolution microscopy techniques to bypass the inherent spatial resolution limitations of DNA FISH. Furthermore, the FRET-FISH probe design described here could be easily adapted to detect DNA-RNA and RNA-RNA interactions in single cells. Lastly, FRET-FISH probes carrying FRET 
donor dyes could be combined with antibodies labeled with FRET acceptor dyes to detect DNA-protein or RNA-protein interactions, instead of using proximity-ligation assays.

Our finding that FRET-FISH measurements correlate stronger with Hi-C than ATAC-seq has important implications for studying chromatin compaction at large scale. Until now, Hi-C has been used mainly to detect 3D genome structural domains, such as TADs and loops, and has not been applied to assess chromatin density. The finding that information about chromatin compaction is contained within $\mathrm{Hi}-\mathrm{C}$ data opens more applications of this powerful technology to measure chromatin compaction at genomic scale across multiple organisms and cell types. In this context, FRET-FISH will be an indispensable companion tool for validating $\mathrm{Hi}-\mathrm{C}$ chromatin density measurements and studying cell-to-cell variability in compaction at selected loci.

In conclusion, we have developed a versatile assay that expands the existing toolkit for studying the spatial organization of the genome in the cell nucleus. Importantly, FRET-FISH could be harnessed not only to probe chromatin compaction at defined genomic regions-which was not possible thus far-but also to study important aspects of structural genome organization such as promoter-enhancer contacts or the formation of condensates. We therefore anticipate that FRET-FISH will be broadly adopted in the genome organization research field.

\section{Methods}

\section{Cell lines}

We obtained mouse embryonic fibroblasts (MEFs) from ATCC (cat. no. SCRC-1040), NIH3T3 fibroblasts from ATCC (cat. no. CRL-1658) and HAP1 chronic myeloid leukemia cells from Horizon Discovery (cat. no. C859). We grew MEFs in Dulbecco's Modified Eagle's Medium (DMEM) (Sigma, cat. no. D6429) supplemented with $15 \%$ fetal bovine serum (FBS) (Sigma, cat. no. F9665), NIH3T3 cells in DMEM supplemented with 10\% FBS, and HAP1 cells in Iscove's Modified Dulbecco's Medium (Sigma, cat. no. 16529) supplemented with $10 \%$ FBS. None of these cell lines is included in the ICLAC database of commonly misidentified cell lines. We regularly checked the cells for Mycoplasma contamination but did not authenticate them. We incubated the cells at $37^{\circ} \mathrm{C}$ in $5 \% \mathrm{O}_{2}$ and $5 \% \mathrm{CO}_{2}$. We grew the cells until they reached $80 \%$ confluency on coverslips (VWR, cat. no. 630-2185) in the case of MEFs and on 9-well chambered coverslips (custom-made by Grace Bio-Labs) in the case of HAP1 cells. We then processed the cells following an adapted version of the $3 \mathrm{D}-\mathrm{FISH}$ protocol ${ }^{30}$, as described in Supplementary

\section{Methods.}

\section{FRET-FISH probe design}

We designed FRET-FISH probes against several genes located along mouse chrX, reasoning that this would allow us to detect differences in chromatin compaction between the active (Xa) and inactive (Xi) homologue in female cells. To select FRET-FISH probe target gene candidates, we used publicly available allelic specific RNA-seq from GEO accession: GSE75659 and ATAC-seq data from GEO accession: 
GSE71156. We used a custom script in MATLAB (R2020a), selection_gene_ATACseq_norm.m, to read the file with normalized ATAC-seq reads assigned to Xa or Xi based on their unique single nucleotide polymorphism (SNP) signature. The probes span regions close to $100 \mathrm{~kb}$, therefore we searched for 110 $\mathrm{kb}$ windows that displayed the largest differences in ATAC-seq read counts between Xa and Xi. The most predominant gene or genes present in the selected windows were compared with the RNA-seq results where high expression levels from Xi means that the gene is an escapee. We aimed at having a balance in between both escapees and inactivated genes.

To design the probes, we created a custom pipeline in MATLAB, that is available at https://github.com/anacmota/FRET-FISH and generates a list of oligonucleotides (oligos) that attain a strict score of uniqueness. The user specifies the oligo length ( () , the number of total oligos $(n)$, the spacing $(s)$ in between consecutive oligomers, the genomic coordinates of the target region and the reference genome against which the oligos must be designed. The genomic sequence of the specified target region is then downloaded from the USCS Genome Browser. In the first MATLAB script, generate_oligos.m, all possible oligo sequences are generated from the target sequence that the user inputs, by moving a sliding window of length / in steps of $1 \mathrm{nt}$ all along the target sequence. Oligos with more than 6 repetitive nucleotides or a GC-content off the 35-80\% range are filtered out from the output list. Secondly, a file named Oligos_list.fa containing the list of oligos remaining after the previous filtering step as well as a variable file named Identities.mat containing all the penalties calculated in the filtering step are generated. The oligos in the Oligos_list.fa list are then run locally through BLASTn (version $2.6 .0+$ ), by setting a threshold of $80 \%$ homology and $80 \%$ mismatches using the following command in Unix:

m blastn -query Oligos_list.fa -db whole_genome.fa -out homologies.txt -evalue 10 -word_size 11 gapopen 15 -gapextend 10 -penalty -3

The parameters of BLASTn are less stringent than in the iFISH pipeline because FRET-FISH probes require a larger number of oligos (typically 450 oligos per probe in FRET-FISH vs. 96 in iFISH) for FRET to be detected. Since the oligo list might be too long for the RAM memory of a common PC, we suggest partitioning it into sub-lists using the following command:

blastn -query Oligos_list.fa -db whole_genome.fa -out homologies.txt -evalue 10 -word_size 11 -gapopen 15 -gapextend 10 -penalty -3

The output file is considerably heavy for posterior analysis, so we suggest removing all the non-relevant information and only keep the oligo name and score using the following command:

awk '\$1 /^Query=/ \{print $\$ 2\} / \wedge$ Score/ \{print $\$ 3\}^{\prime}<$ homologies.txt > homologies.txt.filt

This reduced file is then read line by line by the second MATLAB script Identities_penalty. $m$ and all the homologies found for each oligo are summed up and saved in a MATLAB vector named Scores, which also contains the penalties before BLAST. Subsequently, the calculated homology values are added to the 
file Identities.mat to complete the penalty description for each oligo. The third script, select_best_window.m, searches the best window according to the following parameters specified by the user: 1) space (in nt) in between consecutive oligos carrying the same FRET dye; 2) space in between consecutive oligos carrying different FRET dyes; 3 ) number of oligos in a group of consecutive oligos carrying the same FRET dye; and 4) total number of oligos in the FRET-FISH probe to be designed. The recommended window is computed based on the penalty score of each oligo and the distance distribution in between the oligos. Other parameters can be fine-tuned such as the tolerance for some oligo groups to be incomplete, and slight changes can be made in the distance and homology threshold used to select oligos. The generated oligos are flagged as 'good' when the oligos are within all the thresholds set, whereas they are flagged as 'bad oligos' when they are marked by multiple penalties. An additional filter step is the identification of good oligos whose neighbors are classified as bad and then removed. Lastly, left $(L)$ and right $(R)$ adapter sequences are appended to the 5 ' and 3 ' end, respectively, of each target $(T)$ sequence (see oligo schemes in Fig. $1 \mathrm{~b}$ ) and all the oligo sequences are printed in a text file named result.txt with design parameters information and various summary plots saved in the same folder. For FRET-FISH probes targeting mouse or human genomic regions, 20 nt orthogonal sequences suitable to be used as $\mathrm{F}$ and $\mathrm{R}$ adapters can be found in the list, which we previously described for iFISH (see Supplementary Data 8 in ref. ${ }^{16}$ ). For FRET-FISH probe design 2 and 3 (see Fig. 1b), we added complementary stabilization sequences (SS) to the $3^{\prime}$ (AATTA) and $5^{\prime}$ (TAATT) end of consecutive oligos. We chose sequences composed only of As and Ts since these bases are known to affect fluorescence decay at lower scale compared to $G$ s and $C s$. We purchased all the fluorescently labelled detection oligos ( $L^{\star}$ and $R^{\star}$ in Fig. 1b) from Integrated DNA Technologies (IDT), whereas we produced all the primary oligos composing each FRET-FISH probe starting from synthetic oligopools as described in the

\section{Supplementary Methods.}

\section{Image processing and identification of FRET-FISH dots}

We imaged all the samples using a $100 \times 1.45$ NA objective mounted on a Nikon Eclipse Ti-E inverted microscope system controlled by the NIS Elements software (Nikon) and equipped with an iXON Ultra 888 EMCCD camera (Andor Technology). For each sample, we acquired multiple image stacks, each consisting of 49-70 focal planes spaced $0.3 \mu \mathrm{m}$ apart. A list of filters and dichroic mirrors used in this study is available in Supplementary Table 3. To identify FISH dots, we used our in-house image analysis suite, DOTTER, which is written in MATLAB (MATLAB and Statistics Toolbox Release R2020a) and C99 with GSL (https://www.gnu.org/software/gs//). In DOTTER, cell segmentation is done by thresholding the max intensity projection (in the axial direction). Using watersheds for nuclei separation, the user can then adjust the threshold, in a semi-automatic manner, and tune low- and high-pass filters. To process the FRET-FISH images, we developed a completely automated pipeline in MATLAB, which detects the most intensive signals in each nucleus after segmentation. We acquired images in the donor and acceptor fluorescent channel separately, and only considered FISH dots with similar $x, y, z$ coordinates (radial threshold: 7 pixels) between the donor and acceptor channels. To measure FRET, we excited the donor dye and measured fluorescence in the acceptor channel at the location of each acceptor dot.

\section{FRET efficiency calculation}


In this study we used an emission sensitized FRET assay, so we expected that the FRET intensity would increase when the donor and acceptor dyes are closer in space. Simultaneously, donor fluorescence emission is transferred to the acceptor dye which results in a decrease of donor intensity. Therefore, we calculate the FRET efficiency as following:

$$
F R E T e f f i c i e n c y=\frac{F I_{\text {Acceptor }}}{F I_{\text {Acceptor }}+F I_{\text {Donor }}}
$$

1

where $\mathrm{Fl}_{\text {Acceptor }}$ and $\mathrm{Fl}_{\text {Donor }}$ are the fluorescence intensity measured in the acceptor and donor channel, respectively.

\section{Code availability}

All the MATLAB scripts used to design FRET-FISH probes and to process FRET-FISH images and calculate the FRET efficiency are available at https://github.com/anacmota/FRET-FISH.

\section{Data Availability}

All the raw FRET-FISH images analyzed in this study have been deposited on Figshare and are available at the following DOI: 10.17044/scilifelab.17080892.

(Note: the DOI will be made publicly available only after acceptance of the manuscript for publication. The Reviewers can temporarily access the data at the following private URL:

https://figshare.com/s/f5d96732d1896d952869 .)

A description of all the image datasets generated in this study is available in Supplementary Table 4.

MEFs ATAC-seq and Hi-C data are publicly available on the Gene Expression Omnibus (GEO) database at the following URLs:

- ATAC-seq: https://www.ncbi.nlm.nih.gov/geo/query/acc.cgi?acc=GSE127926

- Hi-C: https://www.ncbi.nlm.nih.gov/geo/query/acc.cgi?acc=GSE76479

\section{Declarations}

\section{Acknowledgements}

We thank Britta Bouwman (Bienko-Crosetto Lab) for critically reading the manuscript and providing valuable comments. This work was supported by grants from the Swedish Research Council (grant no. 2018-02950) and from the Swedish Cancer Society (grant no. CAN 2018/728) to N.C.; and by grants from the Science for Life Laboratory, the Karolinska Institutet KID Funding Program, the Swedish Research 
Council (grant. no. 621-2014-5503), the Human Frontier Science Program (grant. no. CDA-00033/2016-C), the Ragnar Söderberg Foundation (Fellows in Medicine 2016), the Swedish Cancer Society (grant no. 19 $0130 \mathrm{Pj} 03 \mathrm{H}$ ), and the European Research Council under the European Union's Horizon 2020 research and innovation programme (grant no. StG-2016_GENOMIS_715727) to M.B.

\section{Author contributions}

Conceptualization: A.M. Data curation: A.M. Formal analysis: A.M., E.W. Funding acquisition: N.C., M.B. Investigation: A.M., X.W., K.G. Validation: A.M. Methodology. A.M. Project administration: A.M., M.B. Software: A.M., E.W. Supervision: M.B. Visualization: A.M., M.B. Writing: A.M., N.C. and M.B.

\section{Ethics statement}

The authors declare no competing interests.

\section{References}

1. Bannister, A. J. \& Kouzarides, T. Regulation of chromatin by histone modifications. Cell Res. 21, 381395 (2011).

2. Boettiger, A. N. et al. Super-resolution imaging reveals distinct chromatin folding for different epigenetic states. Nature 529, 418-422 (2016).

3. Sexton, T. et al. Three-dimensional folding and functional organization principles of the Drosophila genome. Cell 148, 458-472 (2012).

4. Barski, A. et al. High-resolution profiling of histone methylations in the human genome. Cell 129, 823-837 (2007).

5. Hansen, K. H. et al. A model for transmission of the H3K27me3 epigenetic mark. Nat. Cell Biol. 10, 1291-1300 (2008).

6. Zeng, W., Ball, A. R. \& Yokomori, K. HP1: heterochromatin binding proteins working the genome. Epigenetics 5, 287-292 (2010).

7. Mateos-Langerak, J. et al. Pericentromeric heterochromatin domains are maintained without accumulation of HP1. Mol. Biol. Cell 18, 1464-1471 (2007).

8. Kapuscinski, J. DAPI: a DNA-specific fluorescent probe. Biotech. Histochem. Off. Publ. Biol. Stain Comm. 70, 220-233 (1995).

9. Barth, R., Bystricky, K. \& Shaban, H. A. Coupling chromatin structure and dynamics by live superresolution imaging. Sci. Adv. 6, eaaz2196 (2020).

10. Llères, D., James, J., Swift, S., Norman, D. G. \& Lamond, A. I. Quantitative analysis of chromatin compaction in living cells using FLIM-FRET. J. Cell Biol. 187, 481-496 (2009).

11. Lou, J. et al. Phasor histone FLIM-FRET microscopy quantifies spatiotemporal rearrangement of chromatin architecture during the DNA damage response. Proc. Natl. Acad. Sci. U. S. A. 116, 73237332 (2019). 
12. Song, L. \& Crawford, G. E. DNase-seq: a high-resolution technique for mapping active gene regulatory elements across the genome from mammalian cells. Cold Spring Harb. Protoc. 2010, pdb.prot5384 (2010).

13. Yuan, G.-C. et al. Genome-scale identification of nucleosome positions in S. cerevisiae. Science 309, 626-630 (2005).

14. Buenrostro, J. D., Giresi, P. G., Zaba, L. C., Chang, H. Y. \& Greenleaf, W. J. Transposition of native chromatin for fast and sensitive epigenomic profiling of open chromatin, DNA-binding proteins and nucleosome position. Nat. Methods 10, 1213-1218 (2013).

15. Lieberman-Aiden, E. et al. Comprehensive mapping of long range interactions reveals folding principles of the human genome. Science 326, 289-293 (2009).

16. Gelali, E. et al. iFISH is a publically available resource enabling versatile DNA FISH to study genome architecture. Nat. Commun. 10, 1636 (2019).

17. Kilic, S. et al. Single-molecule FRET reveals multiscale chromatin dynamics modulated by HP1a. Nat. Commun. 9, 235 (2018).

18. Reinius, B. et al. Analysis of allelic expression patterns in clonal somatic cells by single-cell RNA-seq. Nat. Genet. 48, 1430-1435 (2016).

19. Krijger, P. H. L. et al. Cell-of-Origin-Specific 3D Genome Structure Acquired during Somatic Cell Reprogramming. Cell Stem Cell 18, 597-610 (2016).

20. Girelli, G. et al. GPSeq reveals the radial organization of chromatin in the cell nucleus. Nat. Biotechnol. 38, 1184-1193 (2020).

21. Crosetto, N. \& Bienko, M. Radial Organization in the Mammalian Nucleus. Front. Genet. 11, 33 (2020).

22. Visvanathan, A. et al. Modulation of Higher Order Chromatin Conformation in Mammalian Cell Nuclei Can Be Mediated by Polyamines and Divalent Cations. PloS One 8, e67689 (2013).

23. Platani, M., Goldberg, I., Lamond, A. I. \& Swedlow, J. R. Cajal body dynamics and association with chromatin are ATP-dependent. Nat. Cell Biol. 4, 502-508 (2002).

24. Fu, C. et al. Assessment of genetic and epigenetic variation during long-term Taxus cell culture. Plant Cell Rep. 31, 1321-1331 (2012).

25. Simara, P. et al. DNA double-strand breaks in human induced pluripotent stem cell reprogramming and long-term in vitro culturing. Stem Cell Res. Ther. 8, 73 (2017).

26. Murga, M. et al. Global chromatin compaction limits the strength of the DNA damage response. J. Cell Biol. 178, 1101-1108 (2007).

27. Kanda, T., Sullivan, K. F. \& Wahl, G. M. Histone-GFP fusion protein enables sensitive analysis of chromosome dynamics in living mammalian cells. Curr. Biol. CB 8, 377-385 (1998).

28. Chen, X. et al. ATAC-see reveals the accessible genome by transposase-mediated imaging and sequencing. Nat. Methods 13, 1013-1020 (2016).

29. Szabo, Q. et al. Regulation of single-cell genome organization into TADs and chromatin nanodomains. Nat. Genet. 52, 1151-1157 (2020). 
30. Gelali, E. et al. An Application-Directed, Versatile DNA FISH Platform for Research and Diagnostics. Methods Mol. Biol. Clifton NJ 1766, 303-333 (2018).

\section{Figures}

Figure 1

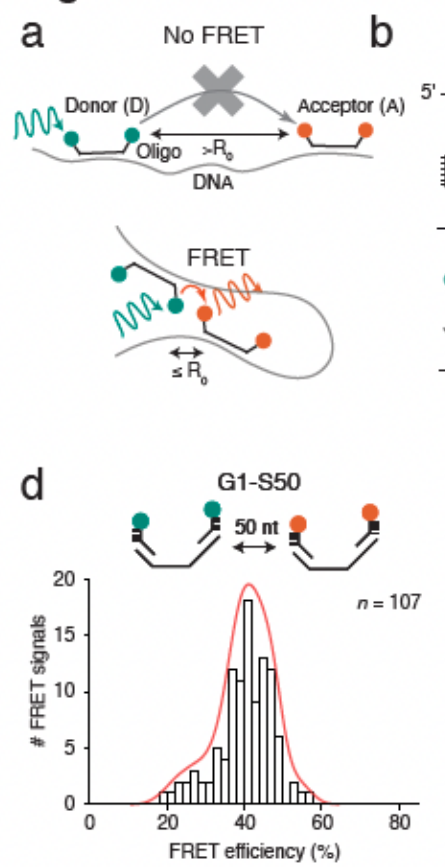

g

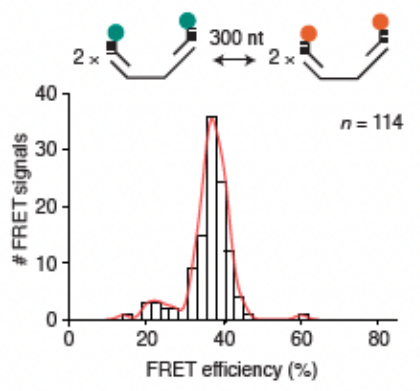

j

Hybridize primary D oligos + both detection oligos

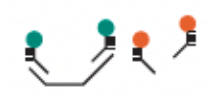

Hybridize primary A oligos + both detection oligos

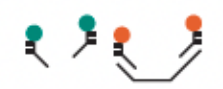

Hybridize primary A+D oligo + both detection oligos

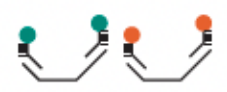

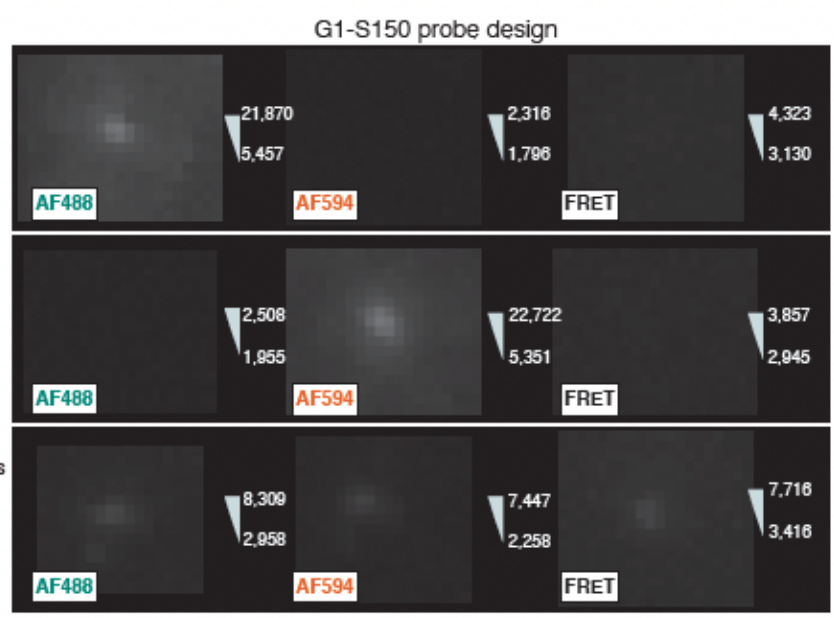

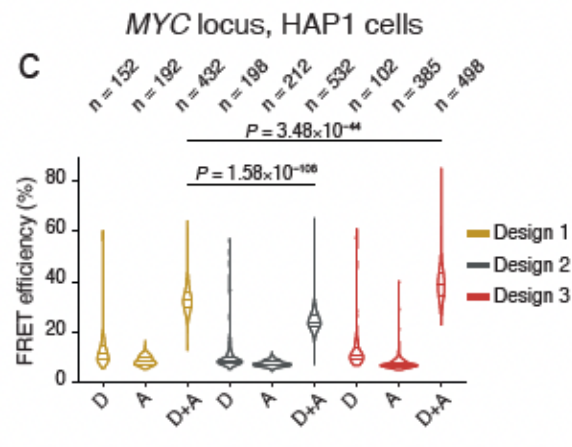

f

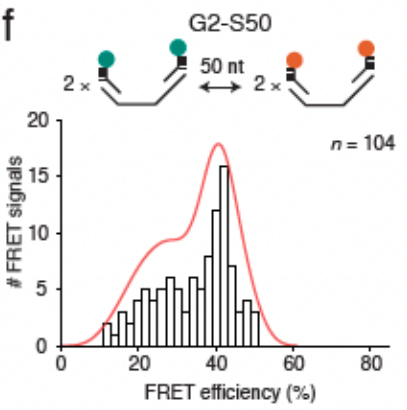

$\mathrm{h}$
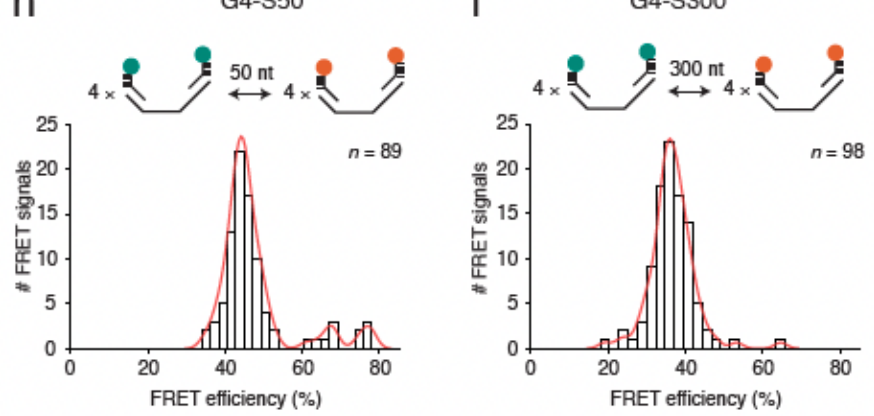

$\mathrm{k}$

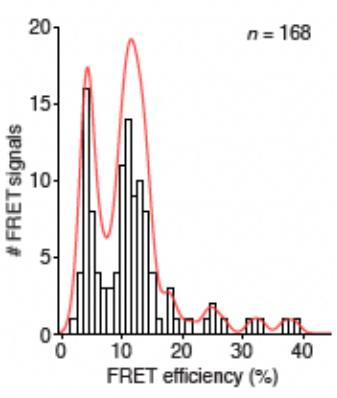

Figure 1 
FRET-FISH implementation and optimization. (a) Schematic representation of how FRET-FISH works. A FRET-FISH probe consisting of alternating oligonucleotides (oligos) labeled with donor (D) or acceptor (A) fluorescent dyes is hybridized to its DNA target in fixed cells. If most of the $D$ and A oligos are farther that the Förster distance, R0, no FRET is detected. In contrast, if a substantial number of D and A oligos are closer that RO, FRET is detected. (b) Scheme of three different FRET-FISH probe designs tested. Each probe is composed of multiple (typically, 200) pairs of donor (D) and acceptor (A) primary oligos containing a oligo sequence complementary to the genomic target $(\mathrm{T})$ flanked by two orthogonal adapter sequences ( $L$ and $R$ ) used as PCR primer docking sites during the production of FRET-FISH probes (see Supplementary Methods) as well as docking sites for complementary detection oligos ( $L^{*}$ and $R^{*}$, respectively) conjugated to FRET donor (green) and acceptor (orange) dyes. In design 2 , the $L$ and $R$ adapter sequences are extended with a $6 \mathrm{nt}$ stabilizing sequence (LSS and RSS, respectively), so that the RSS of one D oligo can anneal with the LSS of the next A oligo. In design 3, the LSS and RSS sequences are added to the $5^{\prime}$ and $3^{\prime}$ end of the $L^{*}$ and $R^{*}$ detection oligos, respectively. (c) Distributions of the FRET efficiency obtained with a FRET-FISH probe targeting the MYC gene in human HAP1 cells, for each of the three probe designs shown in (b). D, probes containing only donor oligos. A, probes containing only acceptor oligos. $D+A$, probes containing both donor and acceptor oligos. $n$, number of FRET signals. $P$, Wilcoxon test, two-tailed. In all the violin plots, the violin extends from the minimum to the maximum value and the horizontal lines represent (from top to bottom) the 75th percentile, the median, and the 25th percentile of the distribution. (d) Distribution of the FRET efficiency obtained with a FRET-FISH probe targeting the Ogt locus in female mouse embryonic fibroblasts (MEFs). The probe is based on design 3 in (b) and consists of multiple groups (G) of one donor (D) and one acceptor (A) oligo spaced (S) $50 \mathrm{nt}$ apart and repeating themselves along between consecutive D and A oligos. Cy3 and Cy5 were used as FRET donor and acceptor dyes, respectively. The orange line represents a kernel density estimation function. $n$, number of FRET signals. (e) Same as in (d), but for a probe in which the $D$ and A oligo in each group are $150 \mathrm{nt}$ apart. (f) Same as in (d), but for a probe in which each group is composed by two $D$ oligos followed by two A oligos. (g) Same as in (f), but for a probe in which the D and A oligo in each group are $300 \mathrm{nt}$ apart. (h) Same as in (d), but for a probe in which each group is composed by four $D$ oligos followed by four A oligos. (i) Same as in (h), but for a probe in which the D and A oligo in each group are 300 nt apart. (j) Example of signal obtained with the probe targeting the mouse Ogt locus shown in (e), hybridizing either only primary D oligos (top) or only primary A oligos (middle) or both (bottom) together with both D and A detection oligos. Alexa Fluor 488 (AF488) and Alexa Fluor 594 (AF594) were used as FRET donor and acceptor dyes, respectively. The white triangles and numbers near each panel represent the minimum (bottom) and maximum (top) intensity values in the corresponding image on the left. (k) Same as in (e) but using AF488 and AF594 as FRET donor and acceptor dyes, respectively. 
Figure 2

MEF cells

a $\quad \operatorname{Atp}_{2 b 3}$ b ${ }_{D d x 3 x} \quad \mathrm{C} \quad K d m 5 c \quad$ d
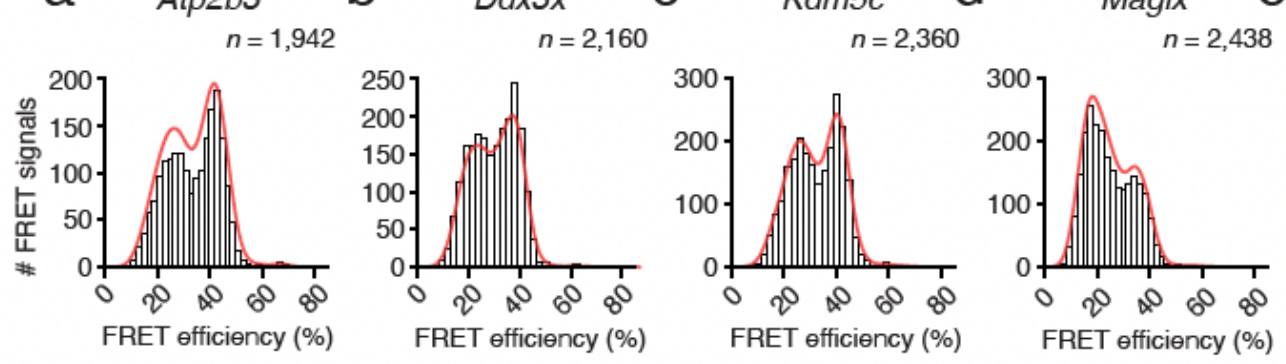

Magix $\mathrm{e}$

Pbdc1 f

Tent5d
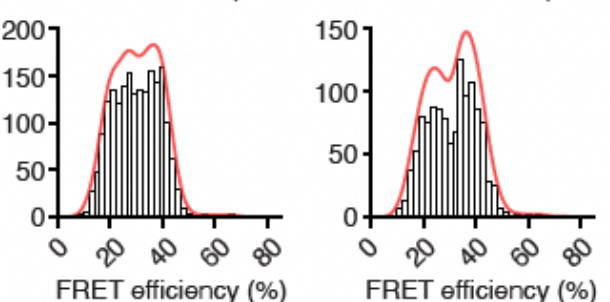

g

$\mathrm{h}$
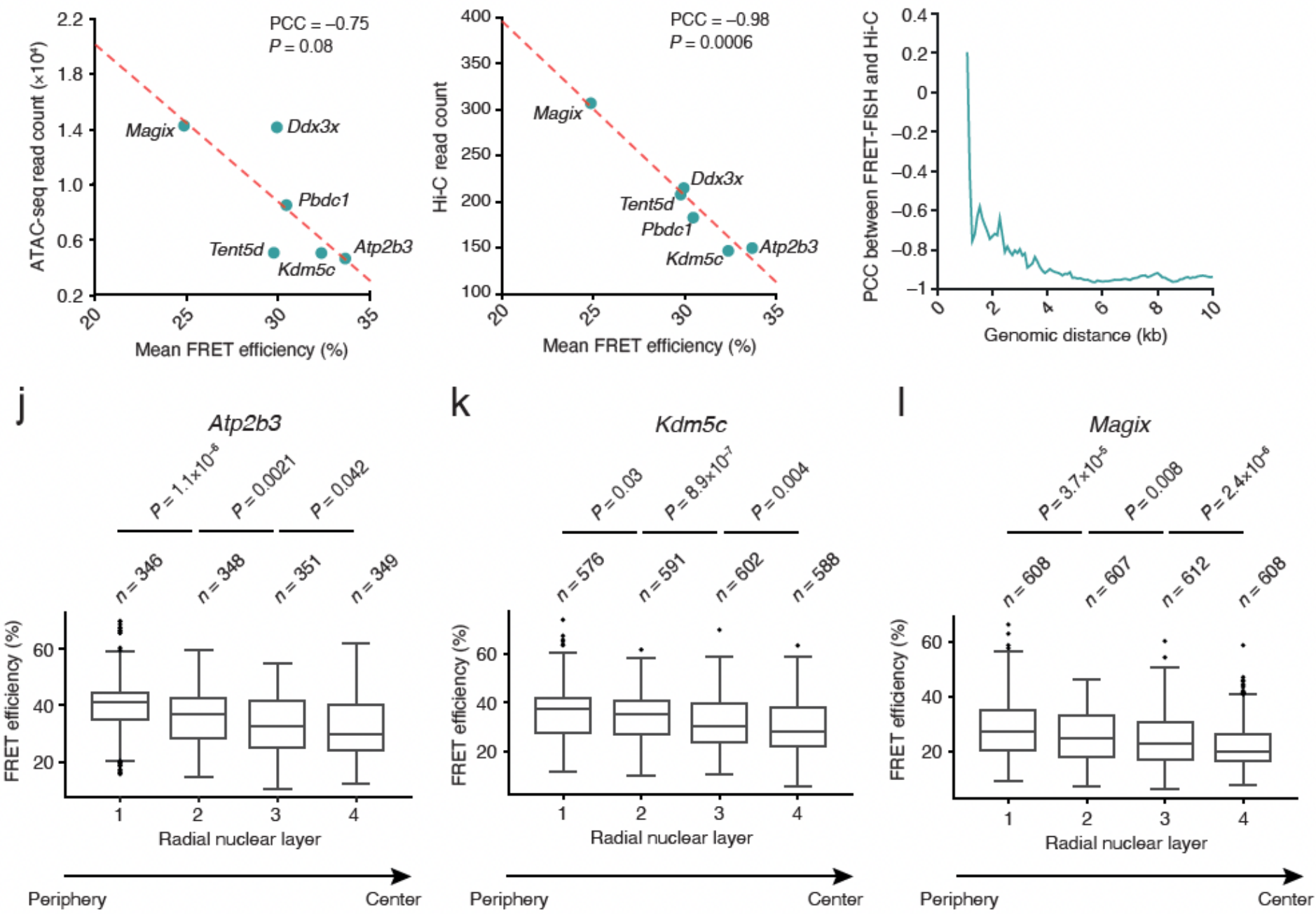

Figure 2

FRET-FISH validation. (a-f) Distributions of FRET efficiencies obtained with FRET-FISH probes targeting six loci on chrX in female mouse embryonic fibroblasts (MEFs). $n$, number of FRET signals obtained in three independent experiments. n, number of FRET signals. The orange line in each plot represents a kernel density estimation function. (g) Correlation between the mean FRET efficiency measured by FRETFISH at the six gene loci shown in (a) and the number of ATAC-seq reads in genomic windows with the same genomic coordinates of the corresponding FRET-FISH probes. PCC, Pearson's correlation 
coefficient. P, Wilcoxon test, two-tailed. The dashed orange line represents the linear regression fit. (h) Same as in (g) but comparing the mean FRET efficiency with the number of Hi-C reads in the same genomic windows. (i) Pearson's correlation coefficient (PCC) between the mean FRET efficiency measured by FRET-FISH at the six loci shown in (a-f) and the number of Hi-C contacts at increasing genomic distance thresholds. The strongest anti-correlation (PCC $=-0.98$, as shown in $(h)$ ) is seen for Hi$\mathrm{C}$ contacts between regions up to $6 \mathrm{~kb}$ apart, encompassing the six gene loci shown in (a-f). (j-l) Distributions of the FRET efficiency measured by FRET-FISH at three of the loci shown in (a-f), after assigning each FRET signal to one of four arbitrary concentric nuclear layers based on its radial distance from the nuclear lamina. Each layer corresponds to a quartile in the distribution of distances of the FRET signals from the nuclear lamina in the corresponding 2D nuclear segmentation masks (see Supplementary Methods). $n$, number of FRET signals. P, Wilcoxon test, two-tailed. In all the boxplots in (jI) and ( $p-r)$, each boxplot extends from the 25th to the 75th percentile, the horizontal bar represents the median, and whiskers extend from $-1.5 \times 1 \mathrm{QR}$ to $+1.5 \times \mathrm{IQR}$ from the closest quartile, where IQR is the interquartile range. Black dots, outliers. 
Figure 3
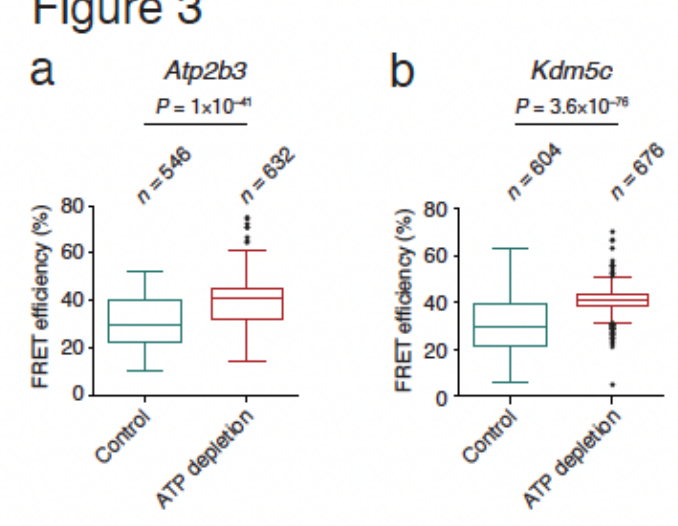

MEF cells

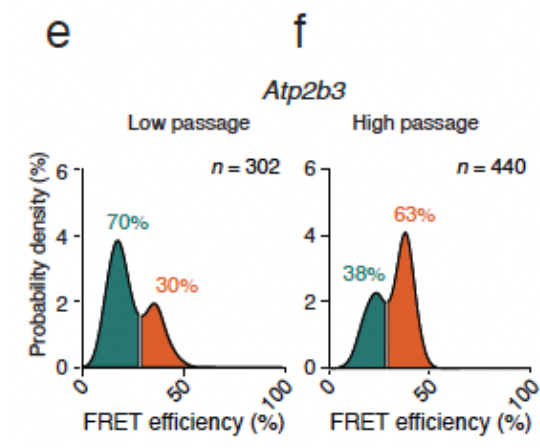

g h
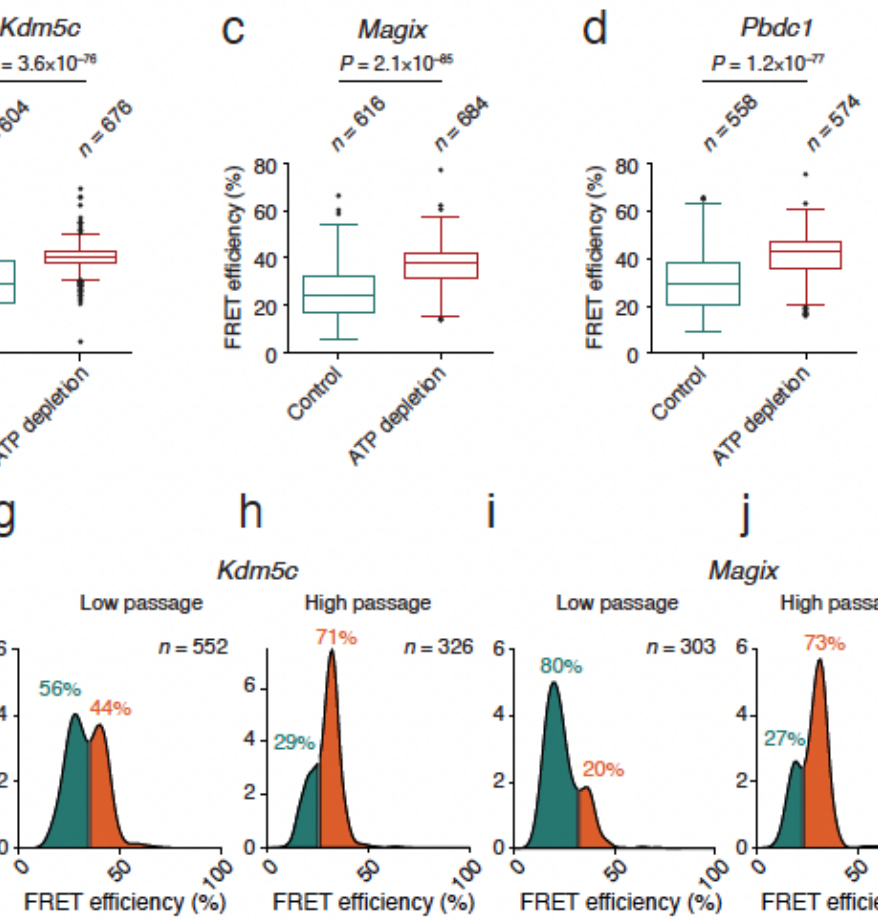

Kdm5c

i
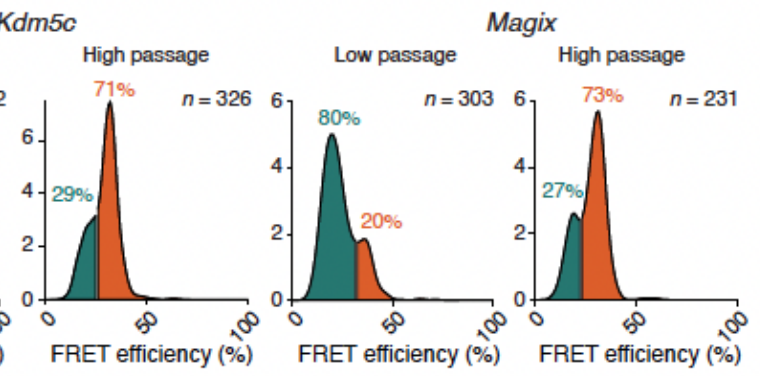

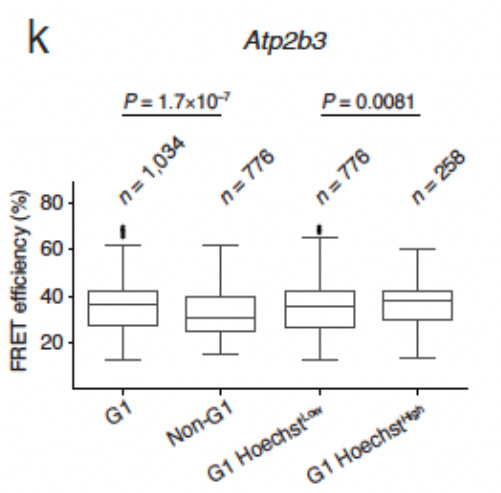

Cell cycle phase

I Kdm5c

$\mathrm{m}$

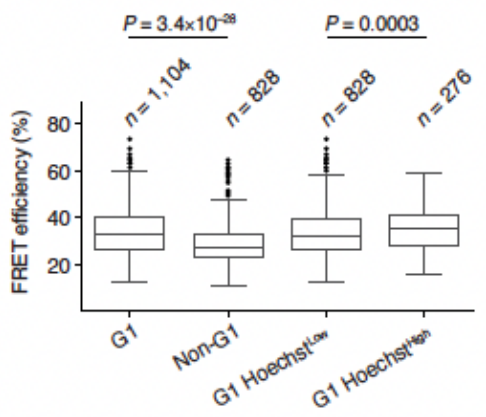

Cell cycle phase

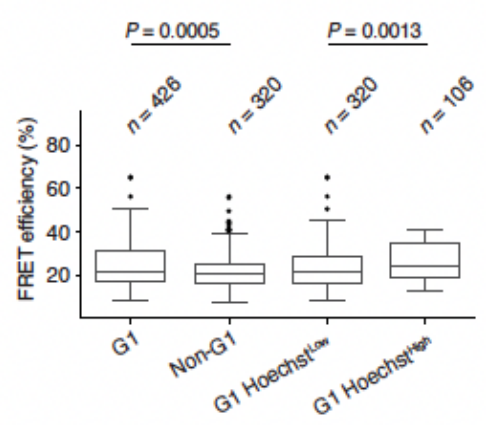

Cell cycle phase $\mathrm{n} \quad \mathrm{O} \quad \mathrm{p}$

Atp2b3 q

Kdm5c
$\mathrm{S}$

Magix

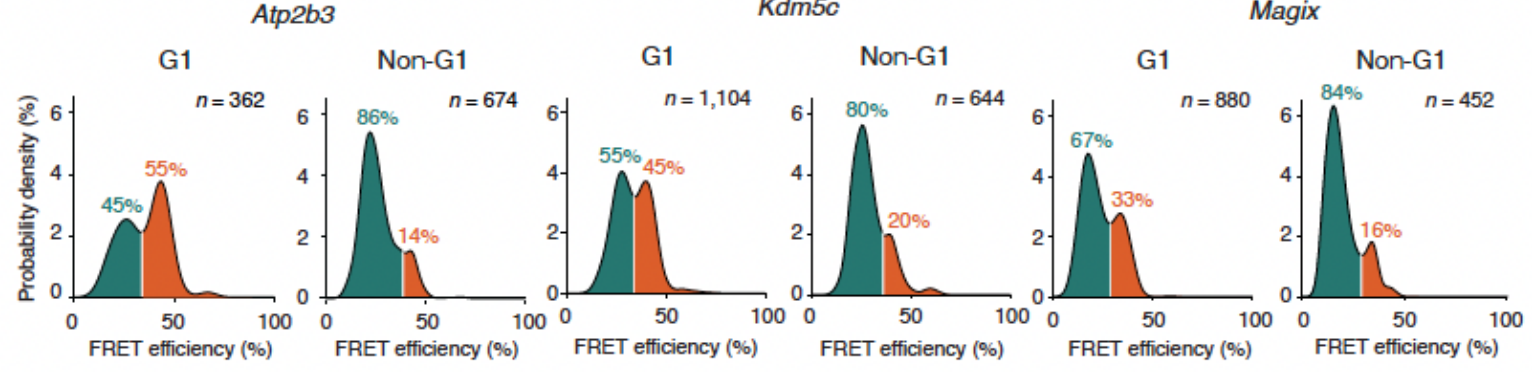

\section{Figure 3}

FRET-FISH detects local chromatin compaction changes reflecting global chromatin condensation changes. (a-d) Distributions of the FRET efficiency measured by FRET-FISH at four different gene loci on mouse chromosome $X$, in female mouse embryonic fibroblasts (MEFs) treated (ATP depletion) or not (control) with a combination of sodium azide and 2-deoxy-d-glucose, which cause ATP depletion and, consequently, global chromatin condensation. n, number of FRET signals. P, Wilcoxon test, two-tailed. (e-j) 
Distributions of the FRET efficiency measured by FRET-FISH at three different gene loci on mouse chrX, in female mouse embryonic fibroblasts (MEFs) at low $(\leq 10)$ or high $(>10)$ in vitro passage numbers. The inflection point in the bimodal distribution was used to automatically distinguish between alleles with a more compact (red) or less compact (green) chromatin state. The percentages on top of each peak indicate the proportion of all the FRET signals assigned to that group. n, number of FRET signals. (k-m) Distributions of the FRET efficiency measured by FRET-FISH at the same loci shown in (e-j) in female MEFs in different phases of the cell cycle. G1 HoechstHigh, G1 cells with values of Hoechst 33342 fluorescence intensity in the nucleus belonging to the top quartile of the corresponding intensity distribution. G1 HoechstLow, G1 cells with values of Hoechst 33342 fluorescence intensity in the nucleus belonging to the first, second and third quartile of the corresponding intensity distribution. n, number of FRET signals. P, Wilcoxon test, two-tailed. (n-s) Distributions of the FRET efficiency measured by FRETFISH at the same loci shown in (e-j) in female MEFs classified as G1 or non-G1 based on their DNA content (see Supplementary Fig. 6a). The inflection point in the bimodal distribution was used to automatically distinguish between alleles with a more compact (red) or less compact (green) chromatin state. The percentages on top of each peak indicate the proportion of all the FRET signals assigned to that group. n, number of FRET signals. In all the boxplots in (a-d) and (k-m), each boxplot extends from the 25th to the 75th percentile, the horizontal bar represents the median, and whiskers extend from $1.5 \times$ IQR to $+1.5 \times$ IQR from the closest quartile, where IQR is the inter-quartile range. Black dots, outliers.

\section{Supplementary Files}

This is a list of supplementary files associated with this preprint. Click to download.

- Supplementarylnformation.pdf

- SupplementaryTable1.xlsx

- SupplementaryTable4.xlsx 